\title{
Jianpi Huashi Tongluo Recipe in the Treatment of Obesity Type 2 Diabetes Mellitus Caused by Damp-heat Trapped Spleen
}

\author{
Xiaohang Tian ${ }^{1}$, Huaizhen Liu ${ }^{2}$ \\ ${ }^{1}$ Graduate School of Anhui University of Traditional Chinese Medicine, Hefei, Anhui, China \\ ${ }^{2}$ The First Affiliated Hospital of Anhui University of Traditional Chinese Medicine, Hefei, Anhui, China
}

\begin{abstract}
Objective: To explore the clinical efficacy of Jianpi Huashi Tongluo Recipe in the treatment of obese patients with type 2 diabetes mellitus caused by damp-heat trapped spleen. Methods: A total of 62 patients who met the inclusion criteria in our hospital from February 2021 to November 2021 were selected as the research subjects, and the treatment methods were selected non-randomly according to their own wishes and conditions. Thirty-one cases in the control group were given subcutaneous injection of liraglutide, and 31 cases in the observation group were given oral Chinese medicine on the basis of the control group. Both groups were treated for 28 consecutive days. The clinical efficacy, TCM syndrome scores, fasting blood glucose (FPG), $2 \mathrm{~h}$ postprandial blood glucose (2hPG), triglyceride (TG), total cholesterol (TC), body mass index (BMI) and waist circumference (WC) were compared between the two groups. Variety. Results: The total effective rate of clinical treatment in the observation group was higher than that in the control group, and the time to reach the blood glucose standard was shorter than that in the control group $(P<0.05)$. The observation group was better than the control group $(P<0.05)$; there was no significant difference in the degree of WC decline between the two groups $(P>0.05)$; the TG and TC of the observation group were better than those of the control group $(P<0.05)$. Conclusion: Jianpi Huashi Tongluo Recipe can effectively control sugar, lose weight, and lower lipids, and can obtain short-term satisfactory curative effect in the treatment of obesity type 2 diabetes mellitus caused by damp-heat trapped spleen.
\end{abstract}

Keywords: Obesity type 2 diabetes mellitus, Damp-heat trapping the spleen, Spleen-invigorating and damp-draining dredging formula, Traditional Chinese Medicine therapy.

\section{Introduction}

The incidence of diabetes mellitus (DM) has increased rapidly worldwide in recent decades, and the incidence of adult type 2 diabetes in China has exceeded 10\% in the past five years[1]. With the adjustment of dietary structure and changes in lifestyle, obesity is becoming more and more popular and younger, and it has become a number of diseases such as type 2 diabetes, stroke, cardiovascular and cerebrovascular diseases, polycystic ovary syndrome, infertility, etc. independent risk factors for the disease. Diabetes and obesity are both metabolic diseases, and the two are closely related. Without timely intervention and effective treatment, diabetes mellitus can easily lead to systemic metabolic dysfunction, various acute and chronic complications, and threaten life. At present, western medicine treatment is mainly based on hypoglycemic drugs, but the indications are limited, and patients who use western medicine for a long time are prone to diarrhea, vomiting and other uncomfortable symptoms, which affect the treatment effect. Professor Liu Huaizhen has been engaged in the clinical practice of traditional Chinese medicine for more than 30 years, and the treatment of this disease with the formula of strengthening the spleen and removing dampness has a definite effect.

\section{Materials and Methods}

\subsection{General Information}

A total of 62 patients who met the inclusion criteria in the First Affiliated Hospital of Anhui University of Traditional Chinese Medicine from February 2021 to November 2021 were selected and randomly divided into two groups according to the patients' conditions and wishes, with 31 cases in each group. In the observation group, there were 14 males and 17 females; age ranged from 20 to 56 years, with an average of $(37.80 \pm 11.41)$ years; weight of 73 to $115 \mathrm{~kg}$, with an average of $(88.37 \pm 11.17) \mathrm{kg}$; the duration of diabetes was 1 month to 13 years, with an average of $(4.45 \pm 11.17) \mathrm{kg} 4.03)$ years. In the control group, there were 15 males and 16 females; aged $20-53$ years, mean $(41.35 \pm 8.92)$ years old; weight $71-112 \mathrm{~kg}$, mean $(89.10 \pm 11.03) \mathrm{kg}$, duration of diabetes mellitus from 1 month to 15 years, mean $(4.86 \pm 11.03)$ $\mathrm{kg}$ 4.14) years. There were no significant differences in age, gender, body weight, and duration of diabetes between the two groups $(\mathrm{P}>0.05)$, which were comparable.

\subsection{Diagnostic Criteria}

\subsubsection{Western Medicine Diagnostic criteria}

The diagnostic criteria for type 2 diabetes refer to the "China Guidelines for the Prevention and Treatment of Type 2 Diabetes (2020 Edition)"[2]: fasting blood glucose $\geq 7.0 \mathrm{mmol} / \mathrm{L}$, or OGTT 2-hour blood glucose $\geq 11.1 \mathrm{mmol} / \mathrm{L}$. The diagnostic criteria for obesity refer to the Guidelines for Primary Diagnosis and Treatment of Obesity (2019)[3]: BMI $\geq 28.0 \mathrm{~kg} / \mathrm{m} 2$ or waist circumference $\geq 90 \mathrm{~cm}$ for men and $\geq 85 \mathrm{~cm}$ for women.

\subsubsection{TCM diagnostic criteria}

Referring to the "Guiding Principles of Clinical Research on New Chinese Medicines (2002 Edition)"[4], it conforms to the syndrome criteria of type 2 diabetes mellitus of type 2 diabetes with damp-heat trapped spleen type: main symptoms: obesity, excessive food and easy hunger; secondary symptoms: fullness in the abdomen and abdomen, limbs 
Drowsiness, pale mouth, not thirsty, yellow urine, sticky stool; tongue pulse: red tongue with yellow greasy coating, slippery pulse.

\subsection{Inclusion and Exclusion Criteria}

\subsubsection{Inclusion criteria}

(1) Meet the above-mentioned Western medicine diagnostic criteria and TCM syndrome differentiation criteria; (2) Age 18 to 60 years old; (3) Normal liver and kidney function.

\subsubsection{Exclusion criteria}

(1) Secondary obesity patients; (2) Use of other weight loss drugs during treatment; (3) Complicated with serious diseases such as heart, brain, liver, and kidney; (4) Pregnant or lactating women; (5) Poor compliance or mental health disease patients.

\subsection{Treatment Methods}

\section{(1) Lifestyle intervention treatment}

(1) Diet: reduce the daily diet by $20 \%-30 \%$; (2) Exercise: perform aerobic exercise 3-5 times a week within the physical tolerance range, each $30-45 \mathrm{~min}$, heart rate $110-150$ times per minute is appropriate; (3) Education: educate patients about basic nutrition knowledge, and encourage patients to learn a healthy lifestyle by themselves.

\section{(2) Control group}

On the basis of lifestyle intervention, subcutaneous injection of liraglutide injection ( $3 \mathrm{ml}: 18 \mathrm{mg} /$ piece, produced by Novo Nordisk (China) Pharmaceutical Co., Ltd., approved by the State Drug Administration J20160037), once a day, 0.6mg/d in the first week, adjusted to $1.2 \mathrm{mg} / \mathrm{d}$ in the second week after adaptation.

\section{(3) Observation group}

On the basis of the control group, Jianpi Huashi Tongluo Recipe was given. The main drug components were: Taizishen 25g, Poria 15g, Atractylodes 15g, Coix seed 20g, Jiang Pinellia 9g, Jiangzhuru 10g, Pueraria lobata 25g, Scutellaria baicalensis $10 \mathrm{~g}$, fried Coptis $6 \mathrm{~g}$, Bupleurum 10g, Salvia 15g, Safflower 15g, Amomum 6g, Gastrodia elata 15g, Zhigancao 6g. Dosage and drugs can be adjusted according to the symptoms. For those with blood stasis, add peach kernel $10 \mathrm{~g}$, for those with qi deficiency, add $20 \mathrm{~g}$ of astragalus, and for those with qi stagnation, add $20 \mathrm{~g}$ of green plum blossom. 1 dose per day, decoct in water, take twice in the morning and evening, $200 \mathrm{~mL}$ each time. Both groups were treated continuously for 28 days.

\subsection{Observation Indicators}

\subsubsection{Biochemical indicators}

Compare FPG, 2hPG, TG, TC, and average blood glucose reaching time before and after treatment.

\subsubsection{Obesity index}

Compare the changes of BMI and WC before and after treatment.

\subsubsection{Efficacy score}

Refer to "Guidelines for Clinical Research on New Chinese Medicines"[4]: the main symptoms are scored as 0, 2, 4, and 6 respectively as none, mild, moderate, and severe; the secondary symptoms are scored as none, mild, moderate, and severe. Score 0, 1, 2, and 3 for each; score 1 for abnormal tongue and pulse, score 0 for normal. A total of 28 points, the lower the score, the milder the symptoms. Markedly effective: symptoms and signs were significantly relieved, fasting or postprandial blood glucose decreased by $\geq 40 \%$, and BMI decreased by $\geq 5 \%$; effective: symptoms and signs were relieved, fasting or postprandial blood glucose decreased by $\geq 20 \%$, and BMI decreased by $2 \%$ to $5 \%$; Invalid: no significant relief or aggravation of symptoms and signs. The total effective rate of treatment $=$ (number of markedly effective cases + number of effective cases)/total number of cases $\times 100 \%$.

\subsection{Statistical Methods}

Data were analyzed and processed by SPSS 26.0 statistical software. The measurement data that obeyed the normal distribution were expressed as $(\bar{x} \pm \mathrm{s})$, the comparison between groups was performed by t test, and the count data was by $\chi 2$ test. $\mathrm{P}<0.05$ was considered statistically significant difference study meaning.

\section{Results}

\subsection{Comparison of Clinical Efficacy Between the Two Groups of Patients}

After treatment, the total effective rate in the observation group was higher than that in the control group, and the difference between the two groups was statistically significant $(\mathrm{P}<0.05)$, as shown in Table 1 .

Table 1: Comparison of clinical efficacy between the two groups of patients (case\%)

\begin{tabular}{|c|c|c|c|c|c|}
\hline Group & Number of cases & Effective & Efficient & Invalid & Always valid \\
\hline Observation group & 31 & $9(29.03)$ & $20(64.52)$ & $2(6.45)$ & $29(93.55)^{1)}$ \\
\hline Control group & 31 & $6(19.35)$ & $17(54.84)$ & $8(25.81)$ & $23(74.19)$ \\
\hline
\end{tabular}

Note: 1) Compared with the control group, $\mathrm{c} 2=4.292, \mathrm{P}=0.038<0.05$.

3.2 Comparison of TCM Syndrome Scores, FPG, 2hPG, BMI, WC, TG, TC Between the Two Groups Before and After Treatment
Before treatment, there was no significant difference in TCM syndrome scores, FPG, 2hPG, BMI, WC, TG and TC between the two groups $(\mathrm{P}>0.05)$. After treatment, the TCM syndrome scores, FPG, 2hPG, and BMI of the two groups were all 
decreased compared with those before treatment, and the observation group was better than the control group $(\mathrm{P}<0.05)$; Statistical significance $(\mathrm{P}>0.05) ; \mathrm{TG}$ and $\mathrm{TC}$ in the observation group were better than those in the control group $(\mathrm{P}<0.05)$, see Table 2.

\subsection{Comparison of the average blood glucose reaching time between the two groups}

The observation group was $7.25 \pm 1.98$ days, and the control group was $9.88 \pm 2.04$ days. The average blood glucose reaching time in the observation group was better than that in the control group $(\mathrm{P}<0.05)$.

\subsection{Comparison of adverse reactions between the two groups}

In the observation group, 2 cases of mild nausea and discomfort occurred during the treatment, and the symptoms improved after symptomatic treatment, which did not affect the treatment. In the control group, 3 cases of nausea and discomfort, 3 cases of loss of appetite and diarrhea occurred during the treatment, which did not affect the subsequent treatment. There was no significant difference in adverse reactions between the two groups $(\mathrm{P}>0.05)$.

Table 2: Comparison of TCM syndrome scores, FPG, 2hPG, BMI, WC, TG, TC between the two groups $(\bar{x} \pm s)$

\begin{tabular}{|c|c|c|c|c|c|c|c|c|c|}
\hline Group & $\begin{array}{l}\text { Number } \\
\text { of cases }\end{array}$ & Time & $\begin{array}{c}\text { TCM } \\
\text { syndrome } \\
\text { score } \\
\end{array}$ & $\begin{array}{c}\text { FPG } \\
(\mathrm{mmol} / \mathrm{L})\end{array}$ & $\begin{array}{c}2 \mathrm{hPG} \\
(\mathrm{mmol} / \mathrm{L})\end{array}$ & $\begin{array}{c}\text { BMI } \\
\left(\mathrm{kg} / \mathrm{m}^{2}\right)\end{array}$ & $\mathrm{WC}(\mathrm{cm})$ & $\begin{array}{c}\mathrm{TG} \\
(\mathrm{mmol} / \mathrm{L})\end{array}$ & $\begin{array}{c}\mathrm{TC} \\
(\mathrm{mmol} / \mathrm{L})\end{array}$ \\
\hline \multirow{2}{*}{$\begin{array}{c}\text { Observation } \\
\text { Group }\end{array}$} & \multirow[b]{2}{*}{31} & Before treatment & $23.40 \pm 1.95$ & $9.09 \pm 2.00$ & $14.48 \pm 4.00$ & $30.74 \pm 2.20$ & $97.23 \pm 6.25$ & $3.07 \pm 1.23$ & $5.02 \pm 1.00$ \\
\hline & & After treatment & $16.35 \pm 2.79^{1) 2)}$ & $5.95 \pm 1.36^{1) 2)}$ & $9.12 \pm 2.89^{1) 2}$ & $\begin{array}{c}28.23 \pm 2.24 \\
1) 2)\end{array}$ & $\begin{array}{c}91.08 \pm 5.87 \\
1)\end{array}$ & $\begin{array}{c}2.00 \pm 0.74 \\
1) 2)\end{array}$ & $\begin{array}{c}4.16 \pm 0.88 \\
1) 2)\end{array}$ \\
\hline \multirow{2}{*}{$\begin{array}{l}\text { Control } \\
\text { Group }\end{array}$} & \multirow[b]{2}{*}{31} & Before treatment & $23.75 \pm 1.80$ & $9.04 \pm 1.76$ & $14.54 \pm 3.84$ & $31.08 \pm 1.85$ & $97.65 \pm 6.71$ & $3.03 \pm 1.62$ & $4.97 \pm 1.03$ \\
\hline & & After treatment & $19.10 \pm 1.77^{1)}$ & $6.89 \pm 1.46^{1)}$ & $10.99 \pm 2.73^{1)}$ & $29.61 \pm 1.72^{1)}$ & $\begin{array}{l}93.25 \pm 5.84 \\
\text { 1) }\end{array}$ & $2.69 \pm 1.26$ & $4.73 \pm 0.86$ \\
\hline
\end{tabular}

Note: 1) Compared with before treatment, $\mathrm{P}<0.05 ; 2)$ Compared with control group, $\mathrm{P}<0.05$.

\section{Discussion}

Diabetes belongs to the category of "quenching thirst" in traditional Chinese medicine. "Simple Questions and Odd Diseases" says: "This overflow of five qi is called spleen deflation. A few foods are sweet and fat. Fat people are hot inside, sweet people are full, and so it overflows and turns into thirst." There is a saying that "fat people have more phlegm and dampness", it can be seen that the spleen is out of transport and transformation. The internal accumulation of phlegm, the heat will damage the body over time, and it will cause thirst. Traditional Chinese medicine believes that diabetes is based on yin deficiency and dryness and heat as the standard, and diagnoses the disease according to the symptoms of "three excesses and one less". With the development of modern medicine, the determination of blood sugar level has become the key to diagnosis, and $80 \%$ of obese patients with type 2 diabetes lack the typical symptoms of "three more and one less". The "three eliminations" dialectical system[5]. Tang Xianyu[6] and others collected and screened literature related to this disease, and statistically concluded that "dampness" is the most common pathological factor in obese patients. The therapeutic effect is not obvious. Based on this, it can be considered that the disease site of obesity type 2 diabetes is centered on the spleen, and phlegm-dampness and yin-deficiency dryness and heat run through the disease. Strengthening the spleen and removing dampness, nourishing yin and clearing heat, removing blood stasis and dredging collaterals are equally important.

The idea of invigorating the spleen and removing dampness and dredging collaterals comes from Gegen Qinlian Quzhuo Recipe and Shenling Baizhu Powder. Gegen Qinlian Quzhuo Decoction is an empirical formula used in our hospital for the treatment of diabetes mellitus with non-alcoholic fatty liver disease. It has a significant long-term effect. It is derived from
Gegen Qinlian Decoction. However, through modern medical research[7], it has been found that it has hypoglycemic effects similar to sulfonylureas and biguanides, and it has insulin sensitization effects in the effective parts of the formula[8] Add phlegm-resolving, blood-stasis-removing and meridiandraining medicines to form Gegen Qinlian Quzhuo Recipe. Combined with Shenling Baizhu Powder, it has the effects of invigorating the spleen and removing dampness, invigorating body fluid and moisturizing dryness, removing blood stasis and dredging collaterals. In the recipe, ginseng is easy to be Taizi ginseng, invigorating the spleen and replenishing qi, preventing ginseng and helping yin damage due to heat; Atractylodes, Poria and Coix seed can invigorate the spleen and infiltrate dampness; Scutellaria baicalensis and Coptis chinensis clear heat and dry dampness; kudzu root removes internal heat in Yangming, promotes Jin and moisturises Dryness; Bupleurum dredging and stagnation, combined with Scutellaria baicalensis can also reconcile Shaoyang; Salvia, safflower promote blood circulation and remove blood stasis; Amomum spleen and dampness; The whole formula has the effects of strengthening the spleen and removing dampness, nourishing yin and clearing away heat, and removing blood stasis and dredging collaterals. Modern pharmacological studies have shown that pueraria (containing puerarin), scutellaria (containing baicalin), and coptis (containing berberine) have excellent performance in lowering blood sugar, improving hyperinsulinemia, regulating lipids, and anti-oxidation[9], Gastrodia[9,10] The active ingredient gastrodin has the functions of expanding blood vessels, enhancing cellular immunity, protecting nerves, etc. It can also prevent diabetic peripheral nerve and peripheral vascular disease.

Liraglutide can induce satiety, delay gastric emptying, reduce food intake, and suppress appetite by activating GLP-1 receptors[11] . In this study, traditional Chinese medicine and liraglutide were used in combination. The results showed that 
the total effective rate of patients treated with combined traditional Chinese and western medicine was higher than that of patients treated with liraglutide alone. TCM syndrome scores, FPG, 2hPG, BMI, TG, TC was better than that of the control group $(\mathrm{P}<0.05)$, and there was no significant difference in the degree of WC decline between the two groups $(\mathrm{P}>0.05)$, but both were lower than those before treatment. It can be seen that for obese patients with type 2 diabetes, especially abdominal obesity, Jianpi Huashi Tongluo Recipe can effectively improve fasting and postprandial blood sugar levels, and can also reduce weight, regulate lipids, and improve clinical efficacy. In addition, the observation period in this study was usually 28 days, which could meet the urgent needs of most patients to control sugar and lose weight, and no obvious adverse reactions occurred in the observation group during the treatment period, indicating that the combination of traditional Chinese and Western medicine in the treatment of obesity type 2 diabetes has high safety.

The disadvantage is that at present, the sample size of this study is small and the observation period is short. The next step is to expand the sample size and prolong the observation time, and conduct more in-depth research on the therapeutic effect of Jianpi Huashi Tongluo Recipe in terms of inflammatory factors.

\section{References}

[1] Wang L, Gao P, Zhang M. et al. Prevalence and ethnic pattern of diabetes and prediabetes in China in 2013[J]. JAMA, 2017, 317(24): 2515-2523.

[2] Diabetes Society of Chinese Medical Association. Guidelines for the prevention and treatment of type 2 diabetes in China (2020 edition)[J]. International Journal of Endocrinology and Metabolism, 2021, 41(05): 482-548.

[3] --. Guidelines for primary diagnosis and treatment of obesity(2019)[J]. Chinese Journal of General Practitioners, 2020(02): 95-101.

[4] State Food and Drug Administration. Guidelines for clinical research of new Chinese medicines[S]. Beijing: China Pharmaceutical Science and Technology Press, 2002: 233-237.

[5] Tong Xiaolin, Bi Guizhi, Zhen Zhong, et al. Classification of TCM syndrome types in 2518 obese patients with type 2 diabetes[J]. World Journal of Integrated Traditional Chinese and Western Medicine, 2008, 03(01): 26-28.

[6] Tang Xianyu, Fan Guanjie, Zhao Xiaohua. Frequency analysis of TCM syndrome types of obesity type 2diabetes mellitus[J]. Clinical Research of Traditional Chinese Medicine, 2013, 5(20): 115-116.

[7] Pan Jingqiang, Han Chao, Liu Huichun, et al. Experimental study on the hypoglycemic effect of Gegen Qinlian Decoction[J]. China Journal of New Drugs, 2000, 09(03): 167-170.

[8] Meng Xiaohong. A comparative study of Gegen Qinlian Decoction and its effective fractions on experimental metabolic syndrome[D]. Heilongjiang University of Traditional Chinese Medicine, 2007.
[9] Xu Chang, Zhong Meng, Ma Zhijie, et al. Modern research progress of Gegen Qinlian Decoction[J]. Jilin Traditional Chinese Medicine, 2015, 35(06): 629-632.

[10] Jin Shanji, Jin Wenlong. Clinical observation on the treatment of diabetic peripheral neuropathy with gastrodin injection[J]. Modern Medicine and Health, 2006, 22(19): 2923.

[11] Du Yanjing, Feng Yufei, Fu Dexing. Pharmacology and clinical research progress of liraglutide[J]. China Journal of New Drugs, 2010, 19(23): 2115-2119+2123.

\section{Author Profile}

Xiaohang Tian (1997-), female, Han nationality, from Qianjiang, Hubei. 2019-level postgraduate student in Internal Medicine of Traditional Chinese Medicine, First Clinical School of Medicine, Anhui University of Traditional Chinese Medicine; research direction: prevention and treatment of endocrine and metabolic diseases with traditional Chinese medicine.

Huaizhen Liu (1964-), female, master, chief physician, doctoral tutor, research direction: prevention and treatment of endocrine and metabolic diseases with traditional Chinese medicine. 